\title{
Pharmacological Approaches to Modifying HDL: More Basic Science to Understand HDL Metabolism is Necessary
}

\author{
Editorial to: “NO-1886 Up-regulates Niemann-Pick C1 protein (NPC1) expression \\ through Liver X Receptor $\alpha$ Signaling Pathway in THP-1 Macrophage-Derived \\ Foam Cells" by Xin Ma et al.
}

\author{
Michael H. Davidson
}

Published online: 12 May 2009

(C) Springer Science + Business Media, LLC 2009

While lowering LDL-C has well established benefits, modification of HDL by pharmacological therapy remains an elusive goal in demonstrating conclusive improvements in cardiovascular outcomes. Part of the challenge in developing therapeutic approaches to HDL is that it is unclear whether increasing HDL-C levels or modifying HDL function or increasing reverse cholesterol transport is (are) the mechanism(s) by which HDL protects against cardiovascular disease. In light of the spectacular failure of torcetrapib, a CETP inhibitor (with toxic off-target effects), it is prudent to better understand the means by which cells utilize HDL to efflux excess cholesterol.

One potentially important target is the nuclear receptor Liver Receptor X (LXR). LXR is a nuclear receptor found in all cells (misnamed because it was originally discovered in liver cells) that regulates the genes involved in cholesterol efflux to protect the cell from cholesterol toxicity. LXR is known to upregulate ABCA1 in response to oxysterols promoting the efflux of free cholesterol into HDL as an acceptor. LXR agonists have been shown to increase reverse cholesterol transport and reduce atherosclerosis in animal models [1].

M. H. Davidson $(\varangle)$

Preventive Cardiology,

The University of Chicago Pritzker School of Medicine,

Chicago, IL, USA

e-mail: michaeldavidson@radiantresearch.com

M. H. Davidson

Radiant Research,

515 North State Street, Suite 2700,

Chicago, IL 60654, USA
Ma et al, in this issue of Cardiovascular Drugs and Therapy, demonstrate that Neumann-Pick $\mathrm{C} 1$ protein (NPC1) is regulated by LXR [2]. NO-1886, which was originally developed as a lipoprotein lipase activator, has been shown to activate LXR and thereby increase NPC1 expression. This effect is abolished in THP-1 macrophages if interference RNA which block LXR transcription is used in conjunction with NO-1886 [3]. Of interest is that NPC-IL1, the sterol transporter that is inhibited by ezetimibe in the intestine, also appears to be regulated by LXR.

LXR, as a therapeutic target, has been difficult due in effect by increasing SREBP1c in the liver promoting triglyceride synthesis and hepatic steatosis. Therefore, developing selective LXR agonists for the macrophage is the key to identify a means to pharmacologically modify LXR to reduce atherosclerosis [4]. Infusing HDL to reduce atherosclerosis has been shown in pilot human data to reduce atheroma volume measured by intravascular ultrasound. However, unless the excess cholesterol is mobilized to be removed from the cell, HDL infusion alone may not be sufficient in the removal of the majority of excess cholesterol that is stimulating the atherosclerotic process. The work by Ma et al. [2] provides important insights into novel therapies that can be used to enhance the ability of cells, especially macrophages, to mobilize the free cholesterol substrate that HDL, as the acceptor, can remove and return to the liver for clearance into bile. HDL physiology appears to be far more complex than LDL metabolism and the more that can be learned regarding the regulation of cellular cholesterol removal, the more likely there will be pharmacological approaches developed that will translate into clinical benefits. 


\section{References}

1. Naik SU, Wang X, Da Silva JS, et al. Pharmacological activation of liver $\mathrm{X}$ receptors promotes reverdse cholesterol transport in vivo. Circulation 2006;113:90-7.

2. Ma X, Hu YW, Mo ZC, et al. NO-1886 up-regulates Niemann-Pick $\mathrm{C} 1$ protein (NPC1) expression through liver $\mathrm{X}$ receptor $\alpha$ signaling pathway in THP-1 macrophage-derived foam cells. Cardiovasc Drugs Ther. 2009;23: This issue. doi:10.1007/s10557-009-6165-8

3. Duval C, Touche V, Tailleux A, et al. Niemann-PickC1like 1 gene expression is down-regulated by LXR activators in the intestine. Biochem Biophys Res Commun 2006;340:125963.

4. Davidson MH, Toth PP. High-density lipoprotein metabolism: potential therapeutic targets. Am J Cardiol 2007;100:n32-40. 